\title{
Investigation of transient heat current from first principles using complex absorbing potential
}

\author{
Zhizhou Yu, ${ }^{1}$ Lei Zhang, ${ }^{1,2}$ Yanxia Xing, ${ }^{3}$ and Jian Wang ${ }^{1, *}$ \\ ${ }^{1}$ Department of Physics and the Center of Theoretical and Computational Physics, The University of Hong Kong, Hong Kong, China \\ ${ }^{2}$ Department of Physics, McGill University, Montreal PQ, Canada H3A $2 T 8$ \\ ${ }^{3}$ Department of Physics, Beijing Institute of Technology, Beijing 100081, China \\ (Received 3 June 2014; revised manuscript received 23 August 2014; published 23 September 2014)
}

\begin{abstract}
We report on a first-principles investigation of transient heat current through molecular devices under steplike pulse of external and gate voltages. Using the nonequilibrium Green's function (NEGF) approach, an exact solution of transient heat current is obtained that goes beyond the wide-band limit. Combining with densityfunctional theory (DFT), we propose a time-dependent NEGF-DFT formalism to study the transient heat current under a steplike pulse for molecular devices from first principles. Anticipating the huge computational cost in the transient regime, we develop an algorithm to speed up the calculation using the complex absorbing potential (CAP). By adding the CAP to replace the Hamiltonian of leads, the effective self-energy of the Green's function becomes independent of energy, allowing analytic calculation of the triple integrations in the exact solution of transient heat current using the theorem of residue. With this linear scaling algorithm, the computational complexity is greatly reduced, and a first-principles calculation of transient heat current of molecular devices becomes possible. As an example, we apply our NEGF-DFT-CAP formalism for a molecular device, the Di-thiol benzene molecule connected by two semi-infinite aluminum leads, and we calculate the transient heat current under an upward gate voltage pulse. The enhancement of heat current is observed.
\end{abstract}

DOI: 10.1103/PhysRevB.90.115428 PACS number(s): 71.15.Mb, 44.05.+e, 85.65.+h, 73.63.-b

\section{INTRODUCTION}

With the rapid development of nanotechnology, various unique quantum phenomena of nanomaterials have been discovered both experimentally and theoretically, leading to a fascinating prospect in the field of nanoelectronics [1-3]. The thermoelectric effect of molecular devices is one of the interesting issues in nanoelectronics due to its potentially wide applications [4-7]. Recently, the stationary Seebeck coefficient was observed in many low-dimensional nanostructures, such as silicon nanowires and carbon nanotubes [8-10]. Therefore, it is important to study the thermoelectric effect, including the heat current of nanosystems, in order to understand how heat energy carries through and is distributed by electronic current flow. In dc transport, the well-known Landauer-Büttiker formalism used for electrical conductance has been successfully extended to thermal transport [11,12]. In nanoelectronics, many theoretical studies focus on the firstprinciples quantum transport properties of molecular devices based on the nonequilibrium Green's function (NEGF) method combined with the density-functional theory (DFT) [13-15], which is a powerful tool in predicting the transport properties of new nanomaterials. Moreover, the quantum transport of a dc steady state calculated using this first-principles NEGFDFT method can give good agreement with experimental data [16-18]. Despite all these efforts, less attention has been paid to time-dependent thermal transport, especially from a first-principles standpoint.

In nanoelectronics, one of the key issues is to understand the response time of nanodevices upon turning them on/off, which is an important performance indicator of nanodevices, and one that has been studied extensively [19-29]. The time-dependent electric current of such a transient process

\footnotetext{
*jianwang@hku.hk
}

has been solved exactly within the wide-band limit (WBL) using the NEGF method by sending a steplike pulse into the system [19]. It has been numerically demonstrated that in order to investigate transient current in nanodevices from a first-principles standpoint, one has to abandon the WBL approach [26]. In fact, an exact solution of the transient electric current beyond the WBL has been obtained [22]. However, it is numerically a big challenge to implement this exact solution within the framework of NEGF-DFT for nanodevices. This is because there is a triple energy integration in the expression of transient electric current. In addition, due to the existence of sharp resonant states, there are many poles in the complex energy plane that are close to the real energy axis, making the convergence of triple integration extremely difficult. Alternatively, iterating the time-dependent Schrödinger equation within time-dependent density functional theory has been proposed, and details of this implementation have been outlined $[21,23]$. An approximated scheme based on the exact NEGF solution has been proposed that reproduced the result of an exactly solvable model beyond the WBL, and it was implemented within NEGF-DFT to calculate the transient current in nanodevices [26]. Very recently, a linear scaling scheme to calculate the time-dependent transient current by combining the complex absorbing potential (CAP) method within the framework of NEGF-DFT has been developed and implemented in first-principles transport calculation in nanodevices, which significantly reduces the computational complexity [30]. With these technical developments, it is conceivable that the first-principles transient calculation of heat current in nanodevices is within reach.

For the time-dependent heat current due to electrons, the transient heat current has been investigated for a quantum dot under an upward pulse of gate voltage within the WBL where the enhanced thermal power was observed [31]. It is desirable to study the transient heat current when the external bias is switched on. More importantly, in order to 
investigate transient heat current from first principles, we need a theoretical formalism going beyond the WBL. Furthermore, how to calculate transient heat current from first principles for molecular devices is another important issue. It is the purpose of this paper to address these issues.

In this paper, we present an exact solution for transient heat current based on NEGF theory that goes beyond the WBL. The transient heat current can be switched on by either external bias or gate voltage in the presence of dc external bias. We have also implemented this theory within the NEGF-DFT-CAP framework, and we developed an algorithm for first-principles calculation of transient heat current in molecular junctions; CAP is the key ingredient of our approach. The idea of CAP was first introduced in the time-dependent wave propagation calculation to eliminate reflection from boundaries [32], and it was then widely used to tackle time-dependent problems in many quantum-mechanical systems [33-36]. Recently, CAP has been used to deal with quantum transport within the NEGF formulation, and it showed an excellent performance in many quantum transport problems $[37,38]$. The basic idea of CAP is to replace the self-energy of the lead by the transmission-free CAP. Then the transport problem of an open system becomes a scattering problem of a finite closed system. Using CAP, we are able to cast the exact solution of heat current into a wideband form (the effective self-energy in the Green's function is energy-independent), allowing us to use the theorem of residue to reduce the triple energy integrations into a single energy integration, thereby significantly speeding up the calculation. We note that for transient electric current, it is enough to calculate the diagonals of a lesser Green's function $G^{<}(t, t)$ in the time domain [21,30]. For the transient heat current, off-diagonals of a lesser Green's function $G^{<}\left(t, t^{\prime}\right)$ must be calculated, making it more difficult to calculate the transient heat current. As an application of this formalism, we have calculated the transient heat current under an upward gate voltage pulse through a molecular device of Di-thiol benzene (DTB) connected by two Al leads. It is found that the transient gate voltage greatly enhances the heat current, in agreement with a previous study on a quantum dot system.

The paper is organized as follows. In Sec. II, we first present the exact solution of time-dependent heat current under the steplike pulse of external bias and gate voltage using the NEGF method, which can be coupled with DFT for first-principles heat transport calculation. Then the CAP method is introduced to solve the transient heat current of molecular devices. In Sec. III, the numerical calculation of transient heat current of an Al-DTB-Al molecular device under an upward gate voltage is presented. Finally, a discussion and conclusion are given in Sec. IV.

\section{THEORETICAL FORMALISM}

\section{A. Exact solution of time-dependent heat current under steplike gate voltage}

For a general two-probe system, the full Hamiltonian reads

$$
\begin{aligned}
H= & \sum_{\mathbf{k} \alpha} \epsilon_{\mathbf{k} \alpha}(t) c_{\mathbf{k} \alpha}^{\dagger} c_{\mathbf{k} \alpha}+\sum_{n} \epsilon_{n}(t) d_{n}^{\dagger} d_{n} \\
& +\sum_{\mathbf{k} \alpha n}\left[V_{\mathbf{k} \alpha n}(t) c_{\mathbf{k} \alpha}^{\dagger} d_{n}+V_{\mathbf{k} \alpha n}^{*}(t) d_{n}^{\dagger} c_{\mathbf{k} \alpha}\right],
\end{aligned}
$$

where the first two terms are Hamiltonians of the isolated leads and the scattering region. The last term describes the coupling between the leads and the scattering region. Here $c_{\alpha}^{\dagger}$ and $d_{n}^{\dagger}$ are the creation operators of the electron on the lead $\alpha$ ( $\alpha=L, R)$ and the central scattering region, respectively; $\epsilon_{n}$ is the energy level of the central scattering region, which depends on time due to the time-dependent gate voltage, while $\epsilon_{\mathbf{k} \alpha}(t)=$ $\epsilon_{\mathbf{k} \alpha}^{(0)}+v_{\alpha}(t)$ is the energy level in lead $\alpha$ in the presence of external bias and $\epsilon_{\mathbf{k} \alpha}^{(0)}$ is the bare energy levels. We will assume $e=\hbar=1$ from now on. We assume that the bias voltage is $v_{\alpha}(t)=v_{0 \alpha}+\tilde{v}_{\alpha}(t)$ and $\tilde{v}_{\alpha}(t)=v_{\alpha} \theta(t)$ while the gate voltage is $V_{g}(t)=v_{g} \theta(t)$ so that the transient behavior can be studied. Here $v_{0 \alpha}$ is the dc bias and $v_{\alpha}$ is the amplitude of the steplike pulse in the lead $\alpha$.

The time-dependent heat current through the lead $\alpha$ is defined as [31]

$$
\left\langle I_{\alpha}^{h}(t)\right\rangle=\left\langle I_{\alpha}^{E}(t)\right\rangle-\mu_{\alpha}(t)\left\langle I_{\alpha}^{e}(t)\right\rangle,
$$

where $\left\langle I_{\alpha}^{E}(t)\right\rangle$ is the energy current, $\left\langle I_{\alpha}^{e}(t)\right\rangle$ is the particle current, and $\mu_{\alpha}(t)=E_{F}+v_{\alpha}(t)$ is the chemical potential of lead $\alpha$.

The energy current is related to the time derivative of the Hamiltonian describing the leads by $I_{\alpha}^{E}=$ $-\dot{H}_{\alpha}$ [39]. Since the Hamiltonian of the lead depends on time explicitly, we make a gauge transformation $U=$ $\exp \left[i \int_{0}^{t} d \tau \sum_{\mathbf{k} \alpha} \tilde{v}_{\alpha}(\tau) c_{\mathbf{k} \alpha}^{\dagger} c_{\mathbf{k} \alpha}\right]$ [40]. The operator $\hat{a}$ and the Hamiltonian will transform accordingly: $\hat{a} \rightarrow U^{\dagger} \hat{a} U$ and $\hat{H} \rightarrow U^{\dagger} \hat{H} U+i\left(\partial_{t} U^{\dagger}\right) U$. After this transformation, the explicit time dependence of $H_{\alpha}$ is eliminated while the hopping strength acquires a phase factor: $\exp \left[i \int_{0}^{t} d \tau \tilde{v}_{\alpha}(\tau)\right]$.

From the Heisenberg equation of motion, $\dot{H}_{\alpha}=-i\left[H_{\alpha}, H\right]$, the average energy current is written as

$$
\left\langle I_{\alpha}^{E}(t)\right\rangle=-2 \operatorname{Re}\left[\sum_{\mathbf{k} n} \epsilon_{\mathbf{k} \alpha}(t) V_{\mathbf{k} \alpha n}(t) G_{n, \mathbf{k} \alpha}^{<}(t, t)\right],
$$

where $G_{n, \mathbf{k} \alpha}^{<}\left(t, t^{\prime}\right) \equiv i\left\langle c_{\mathbf{k} \alpha}^{\dagger}\left(t^{\prime}\right) d_{n}(t)\right\rangle$ and the coupling strength $V_{\mathbf{k} \alpha n}(t)$ depends on time explicitly due to the gauge transformation. The particle current is given by [18]

$$
\left\langle I_{\alpha}^{e}(t)\right\rangle=-2 \operatorname{Re}\left[\sum_{\mathbf{k} n} V_{\mathbf{k} \alpha n}(t) G_{n, \mathbf{k} \alpha}^{<}(t, t)\right] .
$$

From Eq. (2) we have the heat current,

$$
\left\langle I_{\alpha}^{h}(t)\right\rangle=-2 \operatorname{Re}\left[\sum_{\mathbf{k} n}\left(\epsilon_{\mathbf{k} \alpha}^{(0)}-E_{F}\right) V_{\mathbf{k} \alpha n}(t) G_{n, \mathbf{k} \alpha}^{<}(t, t)\right] .
$$

After analytic continuation, the average heat current becomes

$$
\begin{aligned}
I_{\alpha}^{h}(t)= & -2 \operatorname{Re} \int d t_{1} \operatorname{Tr}\left[G^{r}\left(t, t_{1}\right) \breve{\Sigma}_{\alpha}^{<}\left(t_{1}, t\right)\right. \\
& \left.+G^{<}\left(t, t_{1}\right) \breve{\Sigma}_{\alpha}^{a}\left(t_{1}, t\right)\right],
\end{aligned}
$$

where

$$
\breve{\Sigma}_{\alpha}^{\gamma}\left(t_{1}, t\right)=\sum_{\mathbf{k}}\left(\epsilon_{\mathbf{k} \alpha}^{0}-E_{F}\right) \Sigma_{\mathbf{k} \alpha}^{\gamma, 0}\left(t_{1}-t\right) e^{i \int_{t_{1}}^{t} \tilde{v}_{\alpha}(\tau) d \tau},
$$


with $\gamma=r, a,<$ and $\Sigma_{\mathbf{k} \alpha}^{\gamma, 0}\left(t_{1}-t\right)$ being the nonequilibrium self-energy due to the lead in the absence of an ac pulse [41].

Introducing spectral functions,

$$
A_{\alpha}(\epsilon, t)=\int_{-\infty}^{t} d t^{\prime} e^{i \epsilon\left(t-t^{\prime}\right)} G^{r}\left(t, t^{\prime}\right) e^{i \int_{t^{\prime}}^{t} \tilde{v}_{\alpha}(\tau) d \tau}
$$

and

$$
\Psi_{\alpha}(\epsilon, t)=\int_{-\infty}^{t} d t^{\prime} e^{i \epsilon\left(t-t^{\prime}\right)} G^{<}\left(t, t^{\prime}\right) e^{i \int_{t^{\prime}}^{t} \tilde{v}_{\alpha}(\tau) d \tau} .
$$

We then obtain the heat current

$$
\begin{aligned}
\left\langle I_{\alpha}^{h}(t)\right\rangle= & -2 \int \frac{d \epsilon}{2 \pi} \operatorname{Im} \operatorname{Tr}\left\{\left(\epsilon-\mu_{\alpha}\right) \Gamma_{\alpha}(\epsilon)\right. \\
& \left.\times\left[f_{\alpha}(\epsilon) A_{\alpha}(\epsilon, t)+\Psi_{\alpha}(\epsilon, t)\right]\right\} .
\end{aligned}
$$

Since the lesser Green's function satisfies the Keldysh equation,

$$
\begin{aligned}
G^{<}\left(t, t^{\prime}\right)= & \int d t_{1} \int d t_{2} G^{r}\left(t, t_{1}\right) \Sigma^{<}\left(t_{1}, t_{2}\right) G^{a}\left(t_{2}, t^{\prime}\right) \\
= & i \sum_{\alpha} \int \frac{d \epsilon}{2 \pi} e^{-i \epsilon\left(t-t^{\prime}\right)} f_{\alpha}(\epsilon) A_{\alpha}(\epsilon, t) \Gamma_{\alpha}(\epsilon) \\
& \times A_{\alpha}^{\dagger}\left(\epsilon, t^{\prime}\right) e^{i \int_{t}^{t^{\prime}} \tilde{v}_{\alpha}(\tau) d \tau},
\end{aligned}
$$

we have

$$
\begin{aligned}
\Psi_{\alpha}(\epsilon, t)= & i \sum_{\beta} \int \frac{d \epsilon_{1}}{2 \pi} e^{i\left(\epsilon-\epsilon_{1}\right) t} f_{\beta}\left(\epsilon_{1}\right) A_{\beta}\left(\epsilon_{1}, t\right) \Gamma_{\beta}\left(\epsilon_{1}\right) \\
& \times \int_{-\infty}^{t} d t^{\prime} e^{i\left(\epsilon_{1}-\epsilon\right) t^{\prime}} A_{\beta}^{\dagger}\left(\epsilon_{1}, t^{\prime}\right) e^{i \int_{t^{\prime}}^{t}\left[\tilde{v}_{\alpha}(\tau)-\tilde{v}_{\beta}(\tau)\right] d \tau} .
\end{aligned}
$$

Note that we have two types of bias in the system: dc and ac (steplike pulse) biases. The dc bias $v_{0 \alpha}$ is manifested in the Fermi distribution function $f_{\alpha}(E)=f\left(E-v_{0 \alpha}\right)$ while the ac bias appears as a phase factor $\exp \left[i \int_{t^{\prime}}^{t} \tilde{v}_{\alpha}(\tau) d \tau\right]$ in Eqs. (8) and (12). The general solutions of $A_{\alpha}(\epsilon, t)$ and $\Psi_{\alpha}(\epsilon, t)$ for upward and downward steplike pulses as well as a square pulse can be obtained in a similar fashion as Ref. [22]. For instance, for an upward steplike pulse, we have

$$
\begin{aligned}
A_{\alpha}(\epsilon, t)= & \bar{G}^{r}\left(\epsilon+v_{\alpha}\right) \\
& -\int \frac{d \omega}{2 \pi i} \frac{e^{-i(\omega-\epsilon) t} \bar{G}^{r}\left(\omega+v_{\alpha}\right)}{\omega-\epsilon+v_{\alpha}-i 0^{+}}\left[\frac{v_{\alpha}}{\omega-\epsilon-i 0^{+}}\right. \\
& \left.+\left(v_{g}-\sum_{\beta} v_{\beta} Y_{\alpha \beta}(\epsilon, \omega)\right) \tilde{G}^{r}(\epsilon)\right]
\end{aligned}
$$

with

$$
Y_{\alpha \beta}(\epsilon, \omega)=\frac{\tilde{\Sigma}_{\beta}^{r}(\epsilon)-\tilde{\Sigma}_{\beta}^{r}\left(\omega+v_{\alpha}-v_{\beta}\right)}{\epsilon-\omega-v_{\alpha}+v_{\beta}} .
$$

Here, $\tilde{G}^{r}(\omega)=\left[\omega S-\tilde{H}-\tilde{\Sigma}^{r}(\omega)\right]^{-1}$ and $\tilde{H}$ are, respectively, the retarded Green's function and the Hamiltonian of the initial state at $t=0^{-}$, while $\bar{G}^{r}(\omega)=\left[\omega S-\bar{H}-\bar{\Sigma}^{r}(\omega)\right]^{-1}$ and $\bar{H}$ corresponds to the retarded Green's function and the Hamiltonian of the final state at $t=+\infty$, respectively, and $S$ is the overlap matrix if nonorthogonal basis. In the case of an upward steplike pulse, $\tilde{G}^{r}(\omega)$ is the Green's function with the dc bias $v_{0 \alpha}$ applied on both leads, and $\bar{G}^{r}(\omega)$ is the Green's function with the dc bias $v_{0 \alpha}+v_{\alpha}$. The solution of $\Psi_{\alpha}(\epsilon, t)$ is given by

$$
\begin{aligned}
\Psi_{\alpha}(\epsilon, t)= & i \sum_{\beta} \int \frac{d \epsilon_{1}}{2 \pi} e^{i\left(\epsilon-\epsilon_{1}+v_{\alpha}-v_{\beta}\right) t} f_{\beta}\left(\epsilon_{1}\right) A_{\beta}\left(\epsilon_{1}, t\right) \\
& \times \Gamma_{\beta}\left(\epsilon_{1}\right)\left[\frac{i \tilde{G}^{a}\left(\epsilon_{1}\right)}{\epsilon-\epsilon_{1}+i 0^{+}}+B_{\alpha \beta}^{\dagger}\left(\epsilon, \epsilon_{1}, t\right)\right]
\end{aligned}
$$

with

$$
\begin{aligned}
B_{\alpha \beta}\left(\epsilon, \epsilon_{1}, t\right)= & \operatorname{expc}\left(\epsilon-\epsilon_{1}+v_{\alpha}-v_{\beta} \mid t\right) \bar{G}^{r}\left(\epsilon_{1}+v_{\beta}\right) \\
& -\int \frac{d \omega}{2 \pi i} \frac{\operatorname{expc}\left(\epsilon-\omega+v_{\alpha}-v_{\beta} \mid t\right)}{\omega-\epsilon_{1}+v_{\beta}-i 0^{+}} \\
& \times \bar{G}^{r}\left(\omega+v_{\beta}\right)\left[\frac{v_{\beta}}{\omega-\epsilon_{1}-i 0^{+}}\right. \\
& \left.+\left(v_{g}-\sum_{v} v_{\nu} Y_{\beta \nu}\left(\epsilon_{1}, \omega\right)\right) \tilde{G}^{r}\left(\epsilon_{1}\right)\right],
\end{aligned}
$$

and

$$
\operatorname{expc}(z \mid t)=\frac{e^{i z t}-1}{i\left(z-i 0^{+}\right)}
$$

In the following numerical calculation, we will focus on the case in which $v_{\alpha}=0$ while $v_{g} \neq 0$, i.e., the response of the system when a time-dependent steplike pulse of gate voltage is applied at time $t=0$. In this case, $A_{\alpha}(\epsilon, t)$ and $\Psi_{\alpha}(\epsilon, t)$ do not depend on $\alpha$ and are reduced to

$$
A(\epsilon, t)=\bar{G}^{r}(\epsilon)-v_{g} \int \frac{d \omega}{2 \pi i} \frac{e^{-i(\omega-\epsilon) t}}{\omega-\epsilon-i 0^{+}} \bar{G}^{r}(\omega) \tilde{G}^{r}(\epsilon)
$$

and

$$
\begin{aligned}
\Psi(\epsilon, t)= & i \sum_{\beta} \int \frac{d \epsilon_{1}}{2 \pi} e^{i\left(\epsilon-\epsilon_{1}\right) t} f_{\beta}\left(\epsilon_{1}\right) A\left(\epsilon_{1}, t\right) \Gamma_{\beta}\left(\epsilon_{1}\right) \\
& \times\left[\frac{i \tilde{G}^{a}\left(\epsilon_{1}\right)}{\epsilon-\epsilon_{1}+i 0^{+}}+B^{\dagger}\left(\epsilon, \epsilon_{1}, t\right)\right],
\end{aligned}
$$

where

$$
\begin{aligned}
B\left(\epsilon, \epsilon_{1}, t\right)= & \operatorname{expc}\left(\epsilon-\epsilon_{1} \mid t\right) \bar{G}^{r}\left(\epsilon_{1}\right) \\
& -v_{g} \int \frac{d \omega}{2 \pi i} \frac{\operatorname{expc}(\epsilon-\omega \mid t)}{\omega-\epsilon_{1}-i 0^{+}} \bar{G}^{r}(\omega) \tilde{G}^{r}\left(\epsilon_{1}\right) .
\end{aligned}
$$

In the next section, we will apply this theory to study the transient behavior of heat current for molecular devices from first principles.

\section{B. The calculation of time-dependent heat current under steplike gate voltage within the CAP method}

In this subsection, we present an algorithm to study transient heat current through molecular devices from first principles. To investigate the transient heat current, we have to numerically calculate the Green's function as a function of time. Although the exact solution is available, it is extremely time-consuming to perform the energy integral in Eqs. (10) and (18)-(20) for the 
following reason. It is a triple integral in the energy axis. Due to the existence of the long lifetime of resonant states, many of them are below the Fermi level. There are many poles in the complex energy plane close to the real energy axis, making the convergence of a numerical integral extremely difficult. If we can take the WBL, then the energy integration can be performed analytically since the pole of the Green's function can be found. Since the WBL is an approximation that neglects the band structure of the lead, we wish to go beyond it in this work. In the non-WBL, the poles of Green's function cannot be obtained since the self-energy is a complicated matrix that depends on energy. In view of the success of CAP in the scattering problems, we decide to employ such an approach [30,37]. The basic idea of CAP is to replace the semi-infinite lead by a complex absorbing potential so that there is no reflection at the interface between CAP and the scattering region. Using CAP, the scattering of an open system is mapped to that of a close system of finite size. It has been shown in Ref. [30] that using the energy independence of CAP, the retarded Green's function can be cast into a wide-band form, meaning that the effective self-energy within CAP is energy-independent. This can reduce the numerical calculation of transient properties drastically, as demonstrated in Ref. [30]. To calculate the transient heat current, we use the optimized transmission-free CAP proposed in Ref. [42] as

$$
W(z)=\frac{\hbar^{2}}{2 m}\left(\frac{2 \pi}{\Delta z}\right)^{2} f(z),
$$

with

$$
f(z)=\frac{4}{c^{2}}\left[\left(\frac{\Delta z}{z_{2}-2 z_{1}+z}\right)^{2}+\left(\frac{\Delta z}{z_{2}-z}\right)^{2}-2\right] .
$$

Here $\Delta z=z_{2}-z_{1}$ is the range of CAP along the transport direction $z \cdot z_{1}$ and $z_{2}$ are the staring and ending points of the CAP region, respectively. $c$ is a constant set to be 2.62 in this calculation.

In the first-principles calculation, the total Hamiltonian of the system, including the scattering region and the CAP regions, can be represented by the following matrix:

$$
\left[\begin{array}{ccc}
H_{L L} & H_{L C} & 0 \\
H_{C L} & H_{C C} & H_{C R} \\
0 & H_{R C} & H_{R R}
\end{array}\right]
$$

where, the subscript $L(R)$ and $C$ represent the left (right) CAP region and the central scattering region, respectively. Within the CAP method, the retarded Green's function of lead $\alpha$ can be written as [37]

$$
g_{\alpha \alpha}^{r^{\prime}}(\epsilon)=\left(\epsilon I-H_{\alpha \alpha}^{\prime}\right)^{-1},
$$

where $H_{\alpha \alpha}^{\prime}=H_{\alpha \alpha}-i W_{\alpha}$, and $W_{\alpha}$ is given by Eq. (21). Therefore,

$$
G^{r^{\prime}}(\epsilon)=\left(\epsilon I-H+i \sum_{\alpha} W_{\alpha}\right)^{-1} .
$$

Following Ref. [30], we still use the concept of self-energy and obtain the effective retarded Green's function of the central region within the CAP method as

$$
G_{C C}^{r^{\prime}}(\epsilon)=\left(\epsilon I-H_{C C}+\Sigma^{r^{\prime}}\right)^{-1} .
$$

The self-energy and the linewidth function can be presented as [38]

$$
\begin{gathered}
\Sigma^{r^{\prime}}(\epsilon)=\sum_{\alpha} H_{C \alpha} g_{\alpha \alpha}^{r^{\prime}}(\epsilon) H_{\alpha C}, \\
\Gamma_{\alpha}^{\prime}(\epsilon)=2 H_{C \alpha} g_{\alpha \alpha}^{r^{\prime}}(\epsilon) W_{\alpha} g_{\alpha \alpha}^{a^{\prime}}(\epsilon) H_{\alpha C} .
\end{gathered}
$$

Now the transmission coefficient can be calculated within the CAP method by [38]

$$
T(E)=4 \operatorname{Tr}\left[W_{L}^{\prime} G^{r^{\prime}} W_{R}^{\prime} G^{a^{\prime}}\right] .
$$

Within the NEGF-DFT-CAP formalism, we rewrite the heat current in Eq. (10) as

$$
\begin{aligned}
\left\langle I_{\alpha}^{h}(t)\right\rangle= & -2 \int \frac{d \epsilon}{2 \pi} \operatorname{Im} \operatorname{Tr}\left\{\left(\epsilon-\mu_{\alpha}\right) \Gamma_{\alpha}^{\prime}(\epsilon)\right. \\
& \left.\times\left[f_{\alpha}(\epsilon) A_{C C}^{\prime}(\epsilon, t)+\Psi_{C C}^{\prime}(\epsilon, t)\right]\right\},
\end{aligned}
$$

where

$$
\begin{aligned}
\Psi_{C C}^{\prime}(\epsilon, t)= & 2 i \sum_{\beta} \int \frac{d \epsilon_{1}}{2 \pi} e^{i\left(\epsilon-\epsilon_{1}\right) t} \int_{-\infty}^{t} d t^{\prime} e^{i\left(\epsilon_{1}-\epsilon\right) t^{\prime}} f_{\beta}\left(\epsilon_{1}\right) \\
& \times\left[A^{\prime}\left(\epsilon_{1}, t\right) W_{\beta} A^{\prime \dagger}\left(\epsilon_{1}, t^{\prime}\right)\right]_{C C}
\end{aligned}
$$

Substituting Eqs. (18) and (19) into the above equation, the heat current under the CAP method can be finally written as

$$
\begin{aligned}
\left\langle I_{\alpha}^{h}(t)\right\rangle= & -2 \int \frac{d \epsilon}{2 \pi}\left(\epsilon-\mu_{\alpha}\right) \operatorname{Tr} \operatorname{Im}\left[f_{\alpha}(\epsilon) \Gamma_{\alpha}^{\prime}(\epsilon) A_{C C}^{\prime}(\epsilon, t)\right] \\
& -4 \sum_{\beta} \int \frac{d \epsilon}{2 \pi} \int \frac{d \epsilon_{1}}{2 \pi}\left(\epsilon-\mu_{\alpha}\right) \operatorname{Tr} \\
& \times \operatorname{Re}\left\{e^{i\left(\epsilon-\epsilon_{1}\right) t} f_{\beta}\left(\epsilon_{1}\right) \Gamma_{\alpha}^{\prime}(\epsilon)\right. \\
& \left.\times\left[A^{\prime}\left(\epsilon_{1}, t\right) W_{\beta}\left(\frac{i \tilde{G}^{a^{\prime}}\left(\epsilon_{1}\right)}{\epsilon-\epsilon_{1}+i 0^{+}}+B^{\prime \dagger}\left(\epsilon, \epsilon_{1}, t\right)\right)\right]_{C C}\right\},
\end{aligned}
$$

with

$$
\begin{aligned}
& A^{\prime}(\epsilon, t)=\bar{G}^{r^{\prime}}(\epsilon)-v_{g} \int \frac{d \omega}{2 \pi i} \frac{e^{-i(\omega-\epsilon) t}}{\omega-\epsilon-i 0^{+}} \bar{G}^{r^{\prime}}(\omega) \tilde{G}^{r^{\prime}}(\epsilon) \\
& B^{\prime}\left(\epsilon, \epsilon_{1}, t\right)= \operatorname{expc}\left(\epsilon-\epsilon_{1} \mid t\right) \bar{G}^{r^{\prime}}\left(\epsilon_{1}\right) \\
&-v_{g} \int \frac{d \omega}{2 \pi i} \frac{\operatorname{expc}(\epsilon-\omega \mid t)}{\omega-\epsilon_{1}-i 0^{+}} \bar{G}^{r^{\prime}}(\omega) \tilde{G}^{r^{\prime}}\left(\epsilon_{1}\right)
\end{aligned}
$$

To reduce the triple energy integration in the second term of the heat current into a single integral, we first complete the integration over $\epsilon$ in Eq. (32) using a theorem of residue. To do that, we note that the linewidth function $\Gamma_{\alpha}^{\prime}(\epsilon)$ in Eq. (28) consists of the Green's functions of the lead, which can be 
expanded using their eigenstates as follows:

$$
\begin{aligned}
& g_{\alpha \alpha}^{r^{\prime}}(\epsilon)=\sum_{m} \frac{\left|\psi_{\alpha m}\right\rangle\left\langle\phi_{\alpha m}\right|}{\epsilon-\varepsilon_{\alpha m}}, \\
& g_{\alpha \alpha}^{a^{\prime}}(\epsilon)=\sum_{m} \frac{\left|\phi_{\alpha m}\right\rangle\left\langle\psi_{\alpha m}\right|}{\epsilon-\varepsilon_{\alpha m}^{*}},
\end{aligned}
$$

where the eigenstates are defined as

$$
\begin{aligned}
{\left[H_{\alpha \alpha}-i W\right]\left|\psi_{\alpha m}\right\rangle } & =\varepsilon_{\alpha m}\left|\psi_{\alpha m}\right\rangle, \\
{\left[H_{\alpha \alpha}+i W\right]\left|\phi_{\alpha m}\right\rangle } & =\varepsilon_{\alpha m}^{*}\left|\phi_{\alpha m}\right\rangle .
\end{aligned}
$$

Due to the time-dependent factor $e^{i\left(\epsilon-\epsilon_{1}\right) t}$ in the second term of Eq. (32), the integration of $\Gamma^{\prime}(\epsilon)$ can be done analytically by choosing a complex contour in the upper half-plane. The heat current is simplified as

$$
\begin{aligned}
\left\langle I_{\alpha}^{h}(t)\right\rangle= & -2 \int \frac{d \epsilon}{2 \pi} \operatorname{Tr} \operatorname{Im}\left[\left(\epsilon-\mu_{\alpha}\right) f_{\alpha}(\epsilon) \Gamma_{\alpha}^{\prime}(\epsilon) A_{C C}^{\prime}(\epsilon, t)\right] \\
& -8 \int \frac{d \epsilon}{2 \pi} \operatorname{Tr} \operatorname{Im}\left\{\sum_{\beta} \sum_{m}\left(\varepsilon_{\alpha m}^{*}-\mu_{\alpha}\right) f_{\beta}(\epsilon) H_{C \alpha} g_{\alpha \alpha}^{r^{\prime}}\right. \\
& \times\left(\varepsilon_{\alpha m}^{*}\right) W_{\alpha}\left|\phi_{\alpha m}\right\rangle\left\langle\psi_{\alpha m}\right| H_{\alpha C}\left[A^{\prime}(\epsilon, t) W_{\beta}\right. \\
& \left.\left.\left.\times\left(\frac{i e^{i\left(\varepsilon_{\alpha m}^{*}-\epsilon\right) t} \tilde{G}^{a^{\prime}}(\epsilon)}{\epsilon-\varepsilon_{\alpha m}^{*}}-C^{\prime}\left(\varepsilon_{\alpha m}^{*}, \epsilon, t\right)\right)\right]_{C C}\right\}, \quad 37\right)
\end{aligned}
$$

with

$$
\begin{aligned}
C^{\prime}\left(\varepsilon_{\alpha m}^{*}, \epsilon, t\right)= & \frac{e^{i\left(\varepsilon_{\alpha m}^{*}-\epsilon\right) t}-1}{i\left(\varepsilon_{\alpha m}^{*}-\epsilon\right)} \bar{G}^{a^{\prime}}(\epsilon)-v_{g} \tilde{G}^{a^{\prime}}(\epsilon) \\
& \times \int \frac{d \omega}{2 \pi} \frac{e^{i(\omega-\epsilon) t}-e^{i\left(\varepsilon_{\alpha m}^{*}-\epsilon\right) t}}{\left(\omega-\varepsilon_{\alpha m}^{*}\right)\left(\omega-\epsilon+i 0^{+}\right)} \bar{G}^{a^{\prime}}(\omega) .
\end{aligned}
$$

Note that if we set $\left(\epsilon-\mu_{\alpha}\right)$ and $\left(\varepsilon_{\alpha m}^{*}-\mu_{\alpha}\right)$ equal to 1 in Eq. (37), it will give the expression of the time-dependent electric current under the steplike pulse of gate voltage within the CAP approximation.

The quantity $A^{\prime}(\epsilon, t)$ in Eq. (37) can be calculated analytically using the same method. We first express $\bar{G}^{r^{\prime}}(\epsilon)$ using its eigenstates [30],

$$
\bar{G}^{r^{\prime}}(\epsilon)=\sum_{n} \frac{\left|\Psi_{n}\right\rangle\left\langle\Phi_{n}\right|}{\epsilon-\epsilon_{n}},
$$

where the eigenvalues and eigenstates are defined similar to Eq. (36). After applying the theorem of residue, we find

$$
A^{\prime}(\epsilon, t)=\bar{G}^{r^{\prime}}(\epsilon)-v_{g} \sum_{n} \frac{e^{i\left(\epsilon-\epsilon_{n}\right) t}}{\epsilon-\epsilon_{n}}\left|\Psi_{n}\right\rangle\left\langle\Phi_{n}\right| \tilde{G}^{r^{\prime}}(\epsilon)
$$

and

$$
\begin{aligned}
C^{\prime}\left(\varepsilon_{\alpha m}^{*}, \epsilon, t\right)= & \frac{e^{i\left(\varepsilon_{\alpha m}^{*}-\epsilon\right) t}-1}{i\left(\varepsilon_{\alpha m}^{*}-\epsilon\right)} \bar{G}^{a^{\prime}}(\epsilon)+v_{g} \tilde{G}^{a^{\prime}}(\epsilon) \\
& \times \sum_{n} \frac{e^{i\left(\epsilon_{n}^{*}-\epsilon\right) t}-e^{i\left(\varepsilon_{\alpha m}^{*}-\epsilon\right) t}}{i\left(\epsilon_{n}^{*}-\varepsilon_{\alpha m}^{*}\right)\left(\epsilon_{n}^{*}-\epsilon\right)}\left|\Phi_{n}\right\rangle\left\langle\Psi_{n}\right| .
\end{aligned}
$$

To summarize, in order to calculate the transient heat current under a steplike pulse of gate voltage, we first perform two separate dc calculations using the NEGF-DFT method with and without the gate voltage applied to obtain the Hamiltonian and Green's function of the initial and final states. For the transient calculation, we add the CAP matrix to replace the Hamiltonian of the lead so that our problem becomes the time-dependent evolution of a finite system. To make sure the CAP matrix is accurate enough, we must compare the transmission coefficient of the open system with that obtained from the CAP method. The accuracy can be systematically improved by increasing the length of the CAP region. Once this comparison is done, we can numerically calculate the time-dependent heat current from Eqs. (37), (40), and (41). Since the final expression of transient heat current contains a single energy integral with all the poles calculated, it can be calculated numerically again using the theorem of residue. This makes our method an order $N$ in time, which reduces the computational cost drastically.

Before we end this section, we discuss the advantages and disadvantages of the CAP method. The advantage of the CAP method is its accuracy, which can be improved in a controlled way. In addition, there is no complication implementing the CAP method. The disadvantage of the method is that the system size is increased due to the CAP layer. In this paper, we have used 30 lead units of CAP for left and right buffer regions in order to reproduce the transmission coefficient. We note that the CAP buffer region can be drastically reduced if the CAP is well-designed. For example, there are only six lead units for the calculation of seven-atom monatomic carbon wire sandwiched between Al (100) electrodes in Ref. [37]. Currently, we are still trying to optimize CAP for our atomic basis set. Importantly, the strength of the CAP approach lies in the simulation for a large scattering region. In the large molecular devices that we are aiming to study, there can be a thousand or more atoms in the system. Therefore, comparing with the central molecular device region, the buffer lead region including the CAP region becomes a small portion. Therefore, adding the CAP region does not slow the calculation down very much.

\section{NUMERICAL RESULTS}

In this section, we present the numerical results of the time-dependent heat current response to an upward pulse of gate voltage for the Al-DTB-Al system under a constant dc bias. The two-probe structure of Al-DTB-Al is shown in the inset of Fig. 1. The $\mathrm{Al}(100)$ nanowire is used for the semi-infinite electrode, which is needed for the dc NEGF-DFT calculation for the potential profiles. The distance between the electrode and the sulfur atom is set to be $1.6 \AA$. Our firstprinciples transport calculation is implemented in the NEGFDFT quantum transport package MATDCAL $[43,44]$. The linear combination of atomic orbitals (LCAO) is employed to solve the Kohn-Sham equation. The local density approximation is adopted for the exchange-correlation potential [45], and the nonlocal norm-conserving pseudopotential [46] is used to describe the atomic core. The total energy and Hamiltonian is converged with a tolerance of $10^{-5} \mathrm{eV}$.

For the NEGF-DFT-CAP method, we replace the semiinfinite lead by CAP and leave a buffer region between CAP 


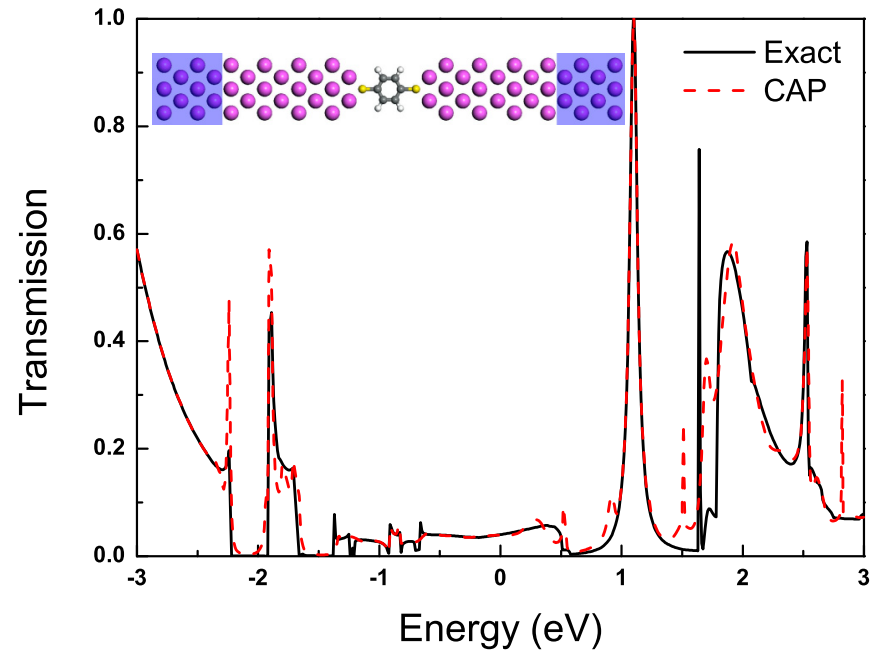

FIG. 1. (Color online) Transmission coefficient of Al-DTB-Al by using the exact method and the CAP method. Inset: Schematic diagram of the two-probe Al-DTB-Al molecular device. The purple shadows present the leads. The pink, gray, yellow, and white balls represent the aluminum, carbon, sulfur, and hydrogen atoms, respectively.

and the DTB molecule. We use 30 unit cells of $\mathrm{Al}(100)$ in the $\mathrm{CAP}$ region along the transport direction. We first compare the transmission coefficient of Al-DTB-Al calculated by the exact method and the CAP method, as shown in Fig. 1. Both cases show that the transmission coefficient is about 0.04 around the Fermi level, which exhibits weak metallic characteristics. Overall, the transmission coefficient calculated from the CAP method shows good agreement with that of the exact method, although the CAP method cannot capture the very sharp transmission peak at $1.65 \mathrm{eV}$. Therefore, we believe that the CAP we constructed is reasonably good.

The time-dependent electric current under the upward steplike gate voltage pulse is then calculated within the firstprinciples NEGF-DFT-CAP framework. Figure 2 shows the

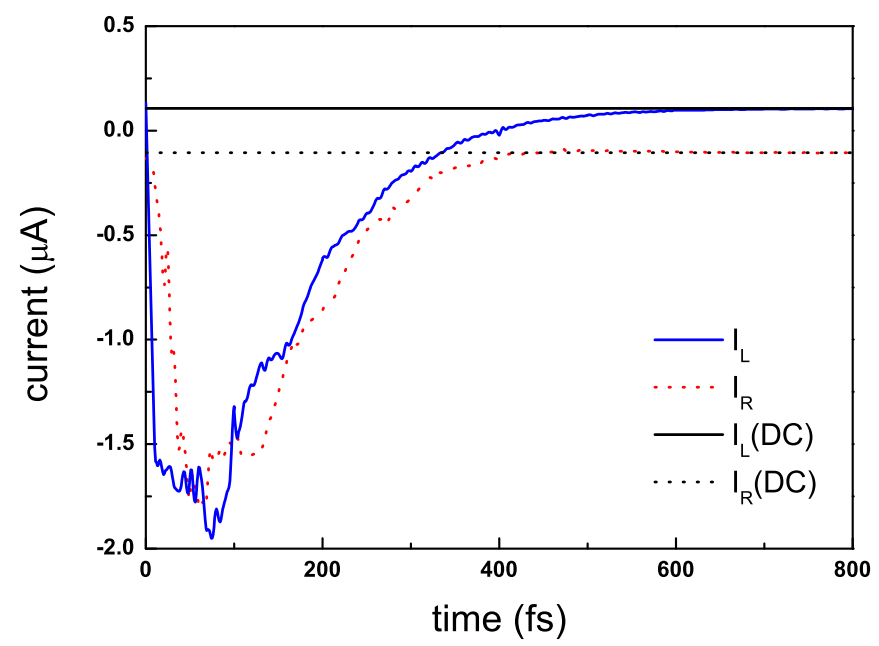

FIG. 2. (Color online) Time-dependent electric current for each lead of Al-DTB-Al under a bias of $0.1 \mathrm{~V}$ with an upward gate voltage pulse of $1 \mathrm{~V}$. transient electric current of Al-DTB-Al under a dc bias voltage of $0.1 \mathrm{~V}\left(v_{0 L}=-v_{0 R}=0.05 \mathrm{~V}\right)$ and an upward transient gate voltage of $1 \mathrm{~V}\left(v_{g}=1 \mathrm{~V}\right)$. The initial current of the left (right) lead without gate voltage is $0.13 \mu \mathrm{A}(-0.13 \mu \mathrm{A})$, so that the dc current is conserved. The switch-on time [48], describing how fast a device can be turned on, is roughly $5 \mathrm{fs}$ for the electric current under the gate voltage pulse. Once the gate voltage switches on, the currents of both the left and the right lead decrease quickly and eventually saturate at a dc limit of $t=+\infty$. Specifically, the current of the left lead changes from positive to negative because the occupied molecular energy levels shift higher than the chemical potential of the left lead due to the sudden increase of the gate voltage. The electrons then flow from those molecular energy levels to the left lead, giving rise to the negative current. After about $80 \mathrm{fs}$, the currents begin to increase again and eventually approach the dc steady-state value that can be calculated from the Landauer-Büttiker formula in the long-time limit, which is $\pm 0.11 \mu \mathrm{A}$ for each lead. The relaxation times [49], namely the elapsed time it takes for the device to achieve a new steady state after the bias is abruptly turned on, are estimated to be about 600 and 420 fs for the transient current of the left and right lead, respectively.

Figure 3 depicts the transient heat current of the Al-DTB-Al molecular junction with the same dc bias and steplike gate voltage as in Fig. 2. The heat current of the initial state is -6.6 and $-6.4 \mathrm{meV} \mu \mathrm{A}$ for the left and right lead, respectively. The difference is the joule heat originating from their different chemical potentials. The transient heat currents of both leads switch on at about $5 \mathrm{fs}$, which is the same as that of the electric current. Then they increase to a high positive value with slight oscillations. The time-dependent heat current of the left lead is always larger than that of the right lead at any time. After about 50 fs, the heat currents reach their maximum values and start to decrease and approach the dc values of the final state calculated within the Landauer-Büttiker formula. Similar to the electric current case, the relaxation time of the heat current of the left lead is longer than that of the right lead, which are

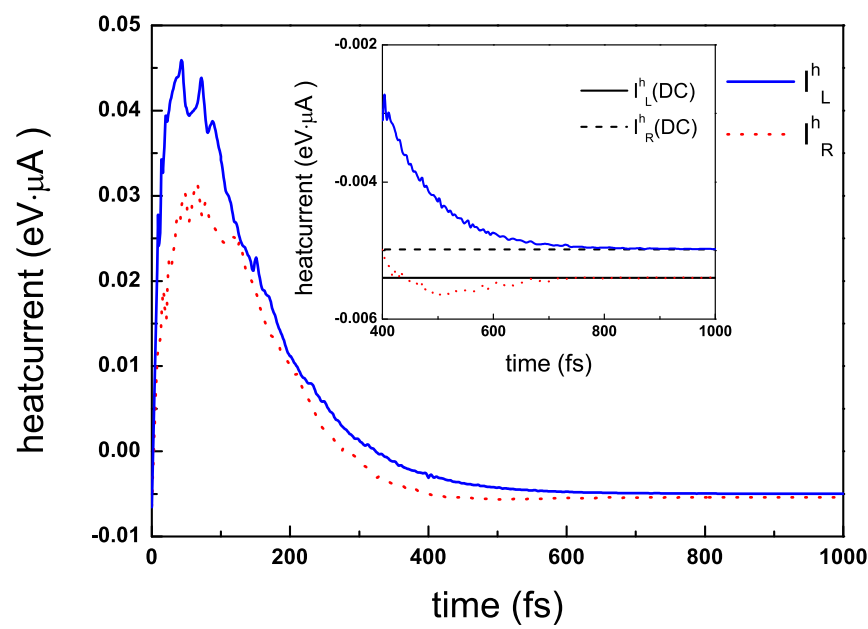

FIG. 3. (Color online) Time-dependent heat current for each lead of Al-DTB-Al under a bias of $0.1 \mathrm{~V}$ with an upward gate voltage pulse of 1 V. Inset: time-dependent heat current compared with the dc steady state in the long-time limit. 
roughly about 810 and 720 fs, respectively. In addition, the heat current needs much more relaxation time than the electric current. The final state of the heat current under the upward gate voltage is about -5.0 and $-5.4 \mathrm{meV} \mu \mathrm{A}$ for the left and right lead, respectively, which is higher than those of the initial state. The difference between the heat current of the left and right lead at the final state is double that of the initial state. It implies a higher Peltier coefficient [47], i.e., the thermopower of this system is enhanced by the gate voltage, which agrees well with previous theoretical research [31].

\section{CONCLUSION}

We have obtained an exact solution for heat current in the presence of a steplike external and gate voltage pulse as well as $\mathrm{dc}$ bias. Our theory is based on NEGF theory and goes beyond the WBL. We have also proposed a first-principles NEGF formalism for transient heat current of molecular devices under the steplike pulse. An algorithm has been developed within the CAP method. By adding the CAP to replace the Hamiltonian of leads, the transport problem of an open system becomes that of a close system. Due to CAP, the non-wide-band problem can be transformed into a wide-band form so that the triple energy integration in the formalism of time-dependent heat current can be calculated using the theorem of residue, which reduces the computational complexity drastically. Our formalism has been applied to study the transient heat current of a molecular junction, Al-DTB-Al molecular device. An enhancement of heat current is observed. Our NEGF-DFT-CAP formalism can also be extended to other transport problems such as steadystate ac heat transport.

Now we discuss the limitation of our formalism. For nonequilibrium quantum transport of charge, the most important quantity is the nonequilibrium density matrix, which is usually obtained using the NEGF-DFT formalism [13]. In this formalism, the density matrix or charge density is constructed at nonequilibrium using the nonequilibrium Green's function within the DFT framework in the dc transport. This procedure applies to the ac case as well except that one has to use the time-dependent DFT (TDDFT). To reduce the computational complexity while still capturing the essential physics, people usually use the adiabatic local density approximation (ALDA) for the exchange and correlation functionals in TDDFT. Recently, the applicability of DFT in open systems has been examined on a rigorous basis by Stefanucci and Almbladh [50]. They have shown that using TDDFT to evolve the density matrix from $\infty$ to time $t$, based on the fundamental RungeGross theorem, gives the same result as that from the NEGFDFT approach.

For transport of heat current, we still used the NEGF-DFT approach where our DFT is implemented using the ALDA approximation for the exchange and correlation functionals in TDDFT. We note that a Runge-Gross-like theorem for the energy density matrix does not yet exist. It is desirable to establish such a theorem to put the NEGF-DFT formalism for heat transport on a more rigorous theoretical footing.

Recently, a formalism has been proposed by Eich et al. [51] to consider heat current due to the external bias and temperature gradient using the NEGF approach, which could be extended for DFT calculation. We note that in the absence of a temperature gradient, their approach is the same as ours.

\section{ACKNOWLEDGMENTS}

This work was financially supported by the Research Grant Council (Grant No. HKU 705212P), the University Grant Council (Contract No. AoE/P-04/08) of the Government of HKSAR, and NSF-China under Grant No. 11374246. Y.X. is supported by NSF-China under Grant No. 11174032.
[1] J. Chen, M. A. Reed, A. M. Rawlett, and J. M. Tour, Science 286, 1550 (1999).

[2] C. Joachim, J. K. Gimzewski, and A. Aviram, Nature (London) 408, 541 (2000).

[3] J. R. Heath and M. A. Ratner, Phys. Today 56(5), 43 (2003).

[4] G. D. Mahan and L. M. Woods, Phys. Rev. Lett. 80, 4016 (1998).

[5] F. J. DiSalvo, Science 285, 703 (1999).

[6] A. Majumdar, Science 303, 777 (2004).

[7] Y. Dubi and M. Di Ventra, Rev. Mod. Phys. 83, 131 (2011).

[8] R. Scheibner, H. Buhmann, D. Reuter, M. N. Kiselev, and L. W. Molenkamp, Phys. Rev. Lett. 95, 176602 (2005).

[9] A. I. Hochbaum, R. Chen, R. D. Delgado, W. Liang, E. C. Garnett, M. Najarian, A. Majumdar, and P. Yang, Nature (London) 451, 163 (2008).

[10] G. U. Sumanasekera, B. K. Pradhan, H. E. Romero, K. W. Adu, and P. C. Eklund, Phys. Rev. Lett. 89, 166801 (2002).

[11] P. N. Butcher, J. Phys. Condens. Matter 2, 4869 (1990).

[12] U. Sivan and Y. Imry, Phys. Rev. B 33, 551 (1986).

[13] J. Taylor, H. Guo, and J. Wang, Phys. Rev. B 63, 245407 (2001).

[14] M. Brandbyge, J.-L. Mozos, P. Ordejón, J. Taylor, and K. Stokbro, Phys. Rev. B 65, 165401 (2002).
[15] A. R. Rocha, V. M. García-Suárez, S. Bailey, C. Lambert, J. Ferrer, and S. Sanvito, Phys. Rev. B 73, 085414 (2006).

[16] C.-C. Kaun, B. Larade, and H. Guo, Phys. Rev. B 67, 121411(R) (2003).

[17] C.-C. Kaun and H. Guo, Nano Lett. 3, 1521 (2003).

[18] T. Frederiksen, M. Brandbyge, N. Lorente, and A.-P. Jauho, Phys. Rev. Lett. 93, 256601 (2004).

[19] N. S. Wingreen, A.-P. Jauho, and Y. Meir, Phys. Rev. B 48, 8487 (1993).

[20] Y. Zhu, J. Maciejko, T. Ji, H. Guo, and J. Wang, Phys. Rev. B 71, 075317 (2005).

[21] S. Kurth, G. Stefanucci, C.-O. Almbladh, A. Rubio, and E. K. U. Gross, Phys. Rev. B 72, 035308 (2005).

[22] J. Maciejko, J. Wang, and H. Guo, Phys. Rev. B 74, 085324 (2006).

[23] G. Stefanucci, S. Kurth, E. K. U. Gross, and A. Rubio, Theor. Comput. Chem. 17, 247 (2007).

[24] G. Stefanucci, S. Kurth, A. Rubio, and E. K. U. Gross, Phys. Rev. B 77, 075339 (2008).

[25] Z. Zhou and S.-I. Chu, Europhys. Lett. 88, 17008 (2009). 
[26] B. Wang, Y. Xing, L. Zhang, and J. Wang, Phys. Rev. B 81, 121103(R) (2010).

[27] S. H. Ke, R. Liu, W. Yang, and H. U. Baranger, J. Chem. Phys. 132, 234105 (2010).

[28] L. Zhang, Y. Xing, and J. Wang, Phys. Rev. B 86, 155438 (2012).

[29] R. Tuovinen, E. Perfetto, G. Stefanucci, and R. van Leeuwen, Phys. Rev. B 89, 085131 (2014).

[30] L. Zhang, J. Chen, and J. Wang, Phys. Rev. B 87, 205401 (2013).

[31] A. Crépieux, F. Šimkovic, B. Cambon, and F. Michelini, Phys. Rev. B 83, 153417 (2011).

[32] R. Kosloff and D. Kosloff, J. Comput. Phys. 63, 363 (1986).

[33] J. G. Muga, J. P. Palao, B. Navarro, and I. L. Egusquiza, Phys. Rep. 395, 357 (2004).

[34] D. E. Manolopoulos, J. Chem. Phys. 117, 9552 (2002).

[35] X.-G. Zhang, K. Varga, and S. T. Pantelides, Phys. Rev. B 76, 035108 (2007).

[36] K. Varga and S. T. Pantelides, Phys. Rev. Lett. 98, 076804 (2007).

[37] J. A. Driscoll and K. Varga, Phys. Rev. B 78, 245118 (2008).

[38] B. G. Cook, P. Dignard, and K. Varga, Phys. Rev. B 83, 205105 (2011).
[39] J.-S. Wang, J. Wang, and N. Zeng, Phys. Rev. B 74, 033408 (2006).

[40] Y. Xing, B. Wang, and J. Wang, Phys. Rev. B 82, 205112 (2010).

[41] Y. Wei and J. Wang, Phys. Rev. B 79, 195315 (2009).

[42] T. Gonzalez-Lezena, E. J. Rackham, and D. E. Manolopoulos, J. Chem. Phys. 120, 2247 (2004).

[43] D. Waldron, P. Haney, B. Larade, A. MacDonald, and H. Guo, Phys. Rev. Lett. 96, 166804 (2006).

[44] D. Waldron, V. Timoshevskii, Y. Hu, K. Xia, and H. Guo, Phys. Rev. Lett. 97, 226802 (2006).

[45] D. M. Ceperley and B. J. Alder, Phys. Rev. Lett. 45, 566 (1980).

[46] N. Troullier and J. L. Martins, Phys. Rev. B 43, 1993 (1991).

[47] J. C. A. Peltier, Ann. Chim. Phys. 56, 371 (1834).

[48] M. Plihal, D. C. Langreth, and P. Nordlander, Phys. Rev. B 61, R13341(R) (2000).

[49] A. Schiller and S. Hershfield, Phys. Rev. B 62, R16271(R) (2000).

[50] G. Stefanucci and C.-O. Almbladh, Europhys. Lett. 67, 14 (2004).

[51] F. G. Eich, A. Principi, M. Di Ventra, and G. Vignale, Phys. Rev. B 90, 115116 (2014). 